\title{
More Harm Than Benefit of Perioperative Dexamethasone on Recovery Following Reconstructive Head and Neck Cancer Surgery : A Prospective Double-Blind Randomized Trial
}

\section{Kainulainen, Satu}

2018-11

Kainulainen , S , Lassus , P , Suominen , A-L , Wilkman , T , Törnwall , J , Thoren , H \& Koivusalo , A-M 2018, ' More Harm Than Benefit of Perioperative Dexamethasone on Recovery Following Reconstructive Head and Neck Cancer Surgery : A Prospective Double-Blind Randomized Trial ' , Journal of Oral and Maxillofacial Surgery, vol. 76 , no. 11 , pp. 2425-2432 . https://doi.org/10.1016/j.joms.2018.05.007

http://hdl.handle.net/10138/305742

https://doi.org/10.1016/j.joms.2018.05.007

draft

Downloaded from Helda, University of Helsinki institutional repository.

This is an electronic reprint of the original article.

This reprint may differ from the original in pagination and typographic detail.

Please cite the original version. 


\section{More Harm Than Benefit of Perioperative Dexamethasone on Recovery Following Reconstructive Head and Neck Cancer Surgery: A Prospective Double-Blind Randomized Trial

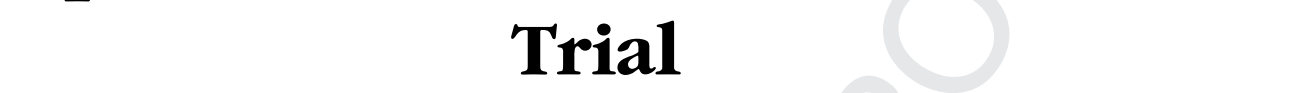 \\ S. Kainulainen, ${ }^{\star} P$. Lassus, $\dagger$ A. L. Suominen, $\ddagger$ T. Wilkman, $\S$ J. Törnwall, $/ /$ H. Thoren, $\$ and A. M. Koivusalo\#}

Purpose: Prospective studies on the effect of dexamethasone after microvascular reconstructive head and neck surgery are sparse despite the widespread use of dexamethasone in this setting. The aim of this study was to clarify whether perioperative use of dexamethasone would improve the quality and speed of recovery. The authors hypothesized that dexamethasone would enhance recovery and diminish pain and nausea.

Materials and Methods: Ninety-three patients with oropharyngeal cancer and microvascular reconstruction were included in this prospective double-blinded randomized controlled trial. Patients in the study group $(n=51)$ received dexamethasone $60 \mathrm{mg}$ over 3 perioperative days; 42 patients did not receive dexamethasone and served as controls. Patient rehabilitation, postoperative opioid and insulin consumption, postoperative nausea and vomiting (PONV), and C-reactive protein (CRP), leukocyte, and lactate levels were recorded.

Results: There was significantly less pain in the study group $(P=.030)$ and the total oxycodone dose for 5 days postoperatively was lower $(P=.040)$. Dexamethasone did not significantly lessen PONV for 5 days postoperatively $(P>.05)$. There were no differences between groups in intensive care unit or hospital stay or in other clinical measures of recovery. Patients receiving dexamethasone required significantly more insulin compared with patients in the control group $(P<.001)$. Lactate and leukocyte levels were significantly higher $(P<.001)$ and CRP levels were significantly lower in the study group.

Conclusion: The only benefit of perioperative dexamethasone use was lower total oxycodone dose; however, the disadvantages were greater. Because dexamethasone can have adverse effects on the

*Consultant, Department of Oral and Maxillofacial Surgery, Helsinki University Hospital, University of Helsinki, Helsinki, Finland.

$\lceil$ Consultant, Department of Plastic Surgery, Helsinki University Hospital, University of Helsinki, Helsinki, Finland.

$\ddagger$ Professor, Institute of Dentistry, University of Eastern Finland, Kuopio; Professor, Department of Oral and Maxillofacial Surgery, Kuopio University Hospital, Kuopio, Finland.

$\S$ Consultant, Department of Oral and Maxillofacial Surgery, Helsinki University Hospital, University of Helsinki, Helsinki, Finland.

\|Consultant, Department of Oral and Maxillofacial Surgery, Helsinki University Hospital, University of Helsinki, Helsinki, Finland.

\Professor, Department of Oral and Maxillofacial Diseases, University of Turku and Turku University Hospital, Turku, Finland.
\#Consultant, Department of Anesthesia and Intensive Care Unit, Helsinki University Central Hospital, Helsinki, Finland

Conflict of Interest Disclosures: None of the authors have a relevant financial relationship(s) with a commercial interest.

Address correspondence and reprint requests to Dr Kainulainen: Department of Oral and Maxillofacial Surgery, Helsinki University Hospital, PO Box 220, FI-00029 HUS, Helsinki, Finland; e-mail: satu.kainulainen@fimnet.fi

Received April 132018

Accepted May 52018

(C) 2018 Published by Elsevier Inc. on behalf of the American Association of Oral and Maxillofacial Surgeons

0278-2391/18/30430-0

hitps://doi.org/10.1016/i.joms.2018.05.007 
postoperative course, routine use of dexamethasone as a pain or nausea medication during reconstructive

head and neck cancer surgery is not recommended.

(C) 2018 Publisbed by Elsevier Inc. on bebalf of the American Association of Oral and Maxillofacial Surgeons

J Oral Maxillofac Surg $\square: 1-8,2018$

Malignant tumors of the head and neck area usually require microvascular reconstruction to repair the surgical defect. Patients often need a tracheostomy, and major postoperative swelling problems are common related side effects from major surgery. Intensive care unit (ICU) and hospital treatment periods are usually long, and anatomically demanding surgery causes major morbidity. ${ }^{1-3}$ Primary healing without postoperative complications is an important goal for surgeons and patients. Prolonged periods of treatment often aggravate postoperative problems and can delay possible adjuvant oncologic treatments.

After a wide variety of surgical procedures, including reconstructive surgery, glucocorticoids (GCs) are given to patients to relieve postoperative pain, swelling, and postoperative nausea and vomiting (PONV), although the evidence of benefit in postoperative use is contentious. ${ }^{4-10}$ In reconstructive head and neck cancer surgery, GCs also are believed to lower the risk of complications, such as prolonged intubation and sedation, and prevent edema in the area of the anastomosis and possible flap loss, thus improving patient recovery. However, even short-term (<1-week) GC use can cause severe complications, ${ }^{11-14}$ wound-healing problems, and postoperative infections. ${ }^{15-17}$ In their previous study, the authors reported that major complications occurred more frequently in patients administered GCs than in the control group $(P=.012)$. In addition, all infections that required surgical intervention within 3 weeks of the operation occurred in patients receiving dexamethasone. ${ }^{18}$

PONV is a common complication after anesthesia and surgery. Emetic episodes can cause numerous complications, such as gastric aspiration, wound dehiscence, psychological distress, and delayed recovery and discharge times. ${ }^{19}$ Especially in reconstructive head and neck surgery, PONV can jeopardize primary healing of the reconstructed area.

The most common complication of GC treatment is an increase in serum glucose concentrations. Increased glucose levels also are believed to influence infections and wound healing. Lactate is a marker of anaerobic metabolism and of perfusion adequacy. Hyperglycemia and hyperlactatemia are associated with increased mortality and are predictors of clinical outcome in patients requiring intensive care. ${ }^{20-22}$ Little has been published on the influence of steroids on lactate levels.
The aims of this study were to clarify the effects of dexamethasone on quality and speed of recovery, pain, PONV, lactate levels, and need for insulin after surgery of patients with microvascular reconstruction for head and neck cancer. The authors hypothesized that dexamethasone would enhance recovery and diminish pain and nausea.

\section{Materials and Methods}

The authors conducted a prospective randomized double-blinded controlled trial involving adult patients with head and neck cancer undergoing reconstructive surgery. The study was performed from December 2008 through February 2013 in the Departments of Oral and Maxillofacial Surgery and Plastic Surgery of the Helsinki University Hospital (Helsinki, Finland). This study followed the Declaration of Helsinki on medical protocol and ethics, and the regional ethical review board of the Helsinki University Central Hospital approved the study. The study was registered with EudraCT (number 2008-000892-11). Written informed consent was obtained from all patients before randomization.

A total of 110 consecutive patients with oropharyngeal cancer who underwent surgery with microvascular reconstruction were included. Patients with the following characteristics were excluded: a history of liver or kidney dysfunction, glaucoma, peptic ulcer, psychosis from the use of steroids, allergy to any constituent of the dexamethasone preparation used (DXM sodium phosphate; Oradexon, N.V. Organon, Netherlands), steroid medication for other diseases, or non-provision of written informed consent.

Patients were randomized into 2 groups; one received perioperative and postoperative dexamethasone (DEX group) and the other did not receive any steroids (controls; NON-DEX group). In the DEX group, patients received dexamethasone $10 \mathrm{mg}$ intravenously (IV) during the induction of anesthesia followed by subsequent 10 -mg doses every 8 hours on the first day, every 12 hours on the second day, and 1 dose on the third day (total, $60 \mathrm{mg}$ ). In the NON-DEX group, patients did not receive dexamethasone. Randomization was performed using sealed envelopes by a person not otherwise involved in the study. The information on which patients would receive dexamethasone was provided in a sealed envelope to the attending anesthesiologist of the operation. 
The same anesthesiologist administered all doses to the patient during the operation and in the ICU postoperatively. Surgeons were unaware of the group to which patients were assigned. The information of the group was not given to the surgeons at any stage during the patient's treatment.

All patients were evaluated by the multidisciplinary head and neck tumor board at the Helsinki University Hospital and were assessed to require free flap reconstruction. Baseline data included demographics, medical history, and information of possible preoperative and postoperative chemotherapy or radiotherapy. Most tumors (92\%) were squamous cell carcinomas. There were 83 fasciocutaneous and 10 osteofasciocutaneus reconstructions. Groups were similar in localizations. The radial forearm was the most frequent flap used $(\mathrm{n}=51)$, followed by the anterolateral thigh flap $(n=32)$. A detailed description of preoperative medical data, surgical data, TNM classifications, parameters during surgery, and complications rated can be found in the authors' previous study. ${ }^{18}$

Surgery was performed under standard balanced anesthesia. Patients were given antibiotics targeted for 7 days, starting with cefuroxime $1.5 \mathrm{~g} \times 3 \mathrm{IV}$ and metronidazole $500 \mathrm{mg} 1 \times 3$ IV on induction of general anesthesia. For allergies, clindamycin $300 \mathrm{mg} \times 4 \mathrm{IV}$ was given. All patients were admitted to the ICU after microvascular reconstruction for the immediate recovery phase. Patients were sedated with a continuous infusion of propofol and alfentanil. After stabilization and verification of the vitality of the microvascular flap, sedation was discontinued and the patient was weaned from the respirator. C-reactive protein (CRP), leukocyte, glucose, and lactate levels were measured for 5 days postoperatively. The targeted glucose level was 5 to $8 \mathrm{mmol} / \mathrm{L}$ and was maintained with insulin infusion; consumption was registered. The total daily amount of insulin was recorded.

Pain was measured using a $10-\mathrm{cm}$ visual analog scale (VAS) of nursing verbal pain scores from 0 (no pain) to 10 (maximum pain) every time before pain medication was administered. All patients received paracetamol $1 \mathrm{~g} \times 3 \mathrm{IV}$ and postoperative pain was controlled using only oxycodone 0.2 to $0.4 \mathrm{mg} / 10 \mathrm{~kg}$ IV. Oxycodone was given if the VAS score was higher than 3. PONV was evaluated whenever patients had severe nausea. The degree of nausea was difficult to grade because of the severity of these operations and most patients were sedated or unable to speak for a long time after surgery. Ondansetron $4 \mathrm{mg}$ IV was administered for PONV if severe retching occurred.

Postoperative data were collected on length of sedation (as duration of propofol infusion), opioid infusion, and intravenous antibiotics. The need for oxycodone and antiemetics was recorded for 5 days postoperatively. Patient rehabilitation (ability to sit, stand, walk, and drink fluids) was recorded. Patients were followed for 30 days after surgery for any surgical or medical complications. Data from the follow-up forms and hospital database were collected and sorted by 1 clinician (S.K.; Table 1).

\section{STATISTICAL ANALYSIS}

The relevance of associations between groups and categorical variables was evaluated by $\chi^{2}$ tests and differences in mean values between groups and continuous variables were evaluated by Wilcoxon 2-sample tests. Differences in pain measured by the VAS and levels of insulin, lactate, and CRP area under the curve (VAS AUC) between groups were assessed by logistic regression.

\section{Results}

\section{RECOVERY}

Ninety-seven patients met the inclusion criteria for the study; 4 patients were excluded. Three patients did not need free flap reconstruction and 1 was accidentally administrated additional dexamethasone. Therefore, the total number of patients included was 93. Of these, 73 were from the department of maxillofacial surgery and 20 were from the department of plastic surgery. There were 51 patients in the DEX group and 42 patients in the NON-DEX group. The size discrepancy between the 2 groups is explained by chance of randomization. No relevant differences were noted in the demographic data between the 2 groups. More patients with diabetes were in the DEX group; this difference was not statistically significant $(P=.116)$. There were no differences between groups in parameters of postoperative mobilization or ability to drink fluids after surgery. There also were no relevant differences in the length of ICU and hospital stay between groups (Table 1).

\section{PAIN}

The total oxycodone dose for 5 days postoperatively was significantly lower in the DEX group than in the NON-DEX group $(P=.040$; Table 1$)$. Patients in the DEX group reported significantly less pain $(P=.030)$ as assessed by the mean VAS AUC measured 7 days postoperatively. The most notable difference in pain was observed during the first postoperative day (Fig 1).

\section{POSTOPERATIVE NAUSEA AND VOMITING}

PONV occurred in 18 patients (19\%) for 5 days postoperatively. Six patients in the DEX group (12\%) received a total dose of ondansetron of $34 \mathrm{mg}$ ( 4 patients received 1 dose and 2 patients received 2 doses); 12 patients in the NON-DEX group (29\%) 
Table 1. PATIENT CHARACTERISTICS AND POSTOPERATIVE RESULTS

393

394

395

396

397

398

399

400

401

402

403

404

405

406

407

408

409

410

411

412

413

414

415

416

417

418

419

420

421

422

423

424

425

426

427

428

429

430

431

432

433

434

435

436

437

438

439

440

441

442

443

444

445

446

447

448

FIGURE 1. VAS area under the curve after microvascular reconstruction, Data are presented as means with $95 \%$ confidence limits. DEX, patients with dexamethasone; NON-DEX, patients without dexamethasone; VAS, visual analog scale.

Kainulainen et al. Dexamethasone and Postoperative Recovery. J Oral Maxillofac Surg 2018. 
was not available from 2 patients ( 1 in the DEX group and 1 in the NON-DEX group) on the second day.

\section{GLUCOSE BALANCE}

Patients in the DEX group required considerably more insulin for 6 postoperative days compared with patients in the NON-DEX group (total insulin needed, 93.5 vs $10.3 \mathrm{U}$, respectively; $P<.001$ ). The greatest difference was on the second postoperative day (Fig 2, Table 1).

\section{METABOLIC AND INFLAMMATORY RESPONSE}

Data on lactate levels were collected only postoperatively. Lactate levels were significantly higher in the DEX group than in the control group $(P<.001)$ for the first 5 postoperative days (Fig 3). CRP levels were significantly lower $(P<.001)$ and leukocyte counts were significantly higher $(P<.001)$ in the DEX group (Figs 4, 5).

\section{Discussion}

The present study showed that dexamethasone had only a minor effect on postoperative healing. Dexamethasone use did not decrease the operation time or the duration of sedation. There was no difference in the length of ICU or hospital stay or the ability to sit, stand, walk, or drink fluids between groups. In their previous study of the same study population, the authors found that the use of dexamethasone was associated with more complications, especially regarding postoperative infections. ${ }^{18}$

Postoperative pain management relieves suffering, can accelerate earlier mobilization, and decrease hospital stay duration and costs. Many published reports in different surgical fields have described the analgesic effect of steroids. ${ }^{23-27}$ In the present study, patients receiving dexamethasone also reported less pain and needed less oxycodone for 5 postoperative days. A study by Clayburgh et $\mathrm{al}^{28}$ showed that extended perioperative corticosteroid use after transoral robotic surgery for initial treatment of oropharyngeal squamous cell carcinoma decreased the length of hospital stay, although postoperative pain was minimally affected. The present trial is the first prospective randomized study to evaluate the perioperative use of dexamethasone in patients with head and neck cancer and microvascular reconstruction. Although the use of dexamethasone decreased the total amount of analgesics and pain, dexamethasone did not accelerate the healing and recovery process and did not shorten the hospital stay.

Dexamethasone is widely used by anesthesiologists to treat PONV. Wattwil et $\mathrm{al}^{29}$ found that ondansetron and dexamethasone were equally effective in the prevention of PONV after breast surgery. Dexamethasone did not meaningfully decrease PONV in the studies of Jahromi et $\mathrm{al}^{30}$ and Furst and Rodarte. ${ }^{31}$ In the present study, dexamethasone did not significantly lessen PONV for 5 days postoperatively $(P>.05)$. Although there was a statistically relevant difference in nausea on the second postoperative day, the clinical difference was not important, because the need for antiemetics was low in the 2 groups (Table 1 ).

In this study, patients who received dexamethasone required considerably more insulin compared with patients who did not receive dexamethasone. In this study, the glucose level was targeted to 5 to $8 \mathrm{mmol} / \mathrm{L}$

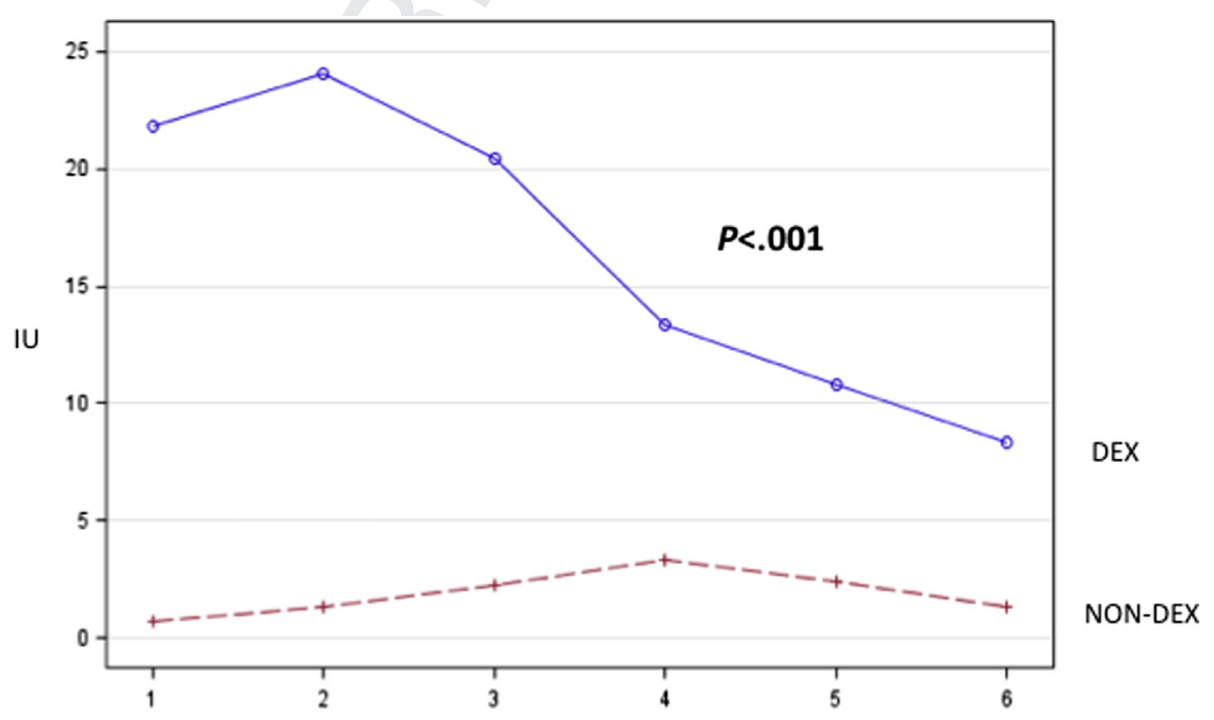

FIGURE 2. Insulin area under the curve after microvascular reconstruction. Data are presented as means with $95 \%$ confidence limits. DEX, patients with dexamethasone; IU, international units of insulin; NON-DEX, patients without dexamethasone. 


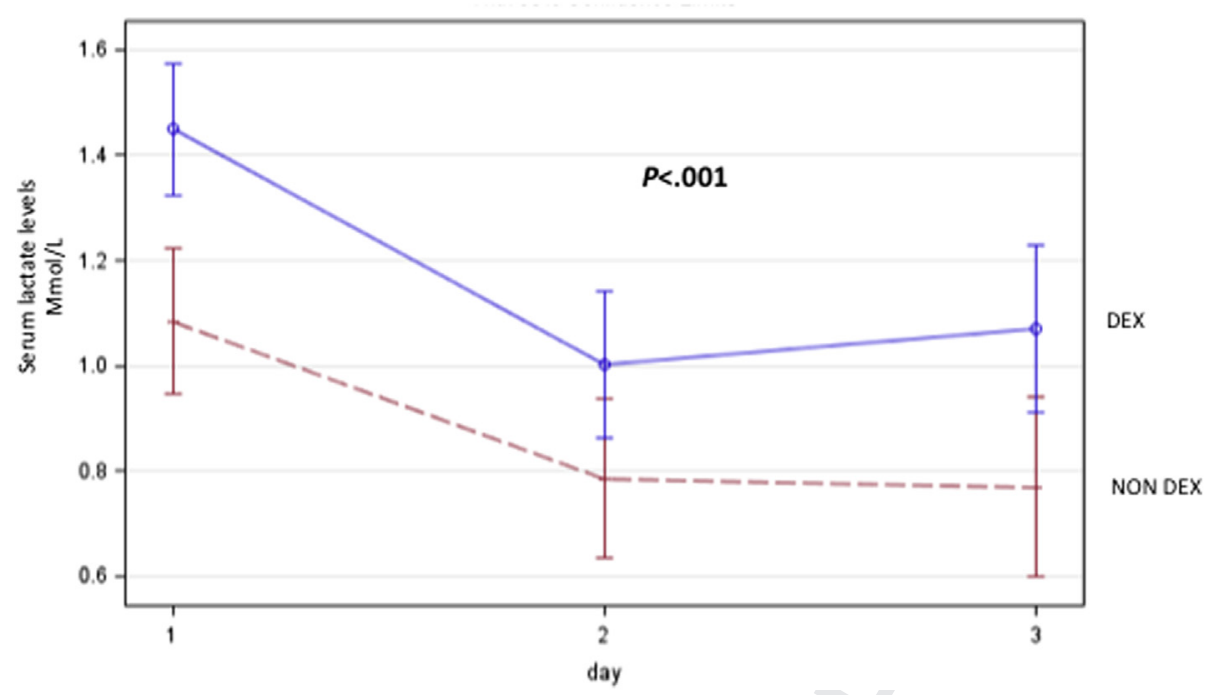

FIGURE 3. Serum or plasma lactate levels after microvascular reconstruction. Data are presented as means with $95 \%$ confidence limits. DEX, patients with dexamethasone; NON-DEX, patients without dexamethasone.

Kainulainen et al. Dexamethasone and Postoperative Recovery. J Oral Maxillofac Surg 2018. and was maintained within these limits with insulin infusions. The authors recorded the total amount of insulin needed but did not collect the data on blood glucose levels. The authors considered this the best method to reflect glucose balance in this setting. The effects of corticosteroids on glucose balance are well known. A multicenter randomized double-blinded placebo-controlled trial of 4,494 patients undergoing cardiac surgery with cardiopulmonary bypass by Dieleman et $\mathrm{al}^{32}$ showed that dexamethasone was associated with higher postoperative glucose levels and that dexamethasone use did not provide patient benefit. The present study showed that accurate glucose monitoring is needed for at least 5 days after surgery, because the need for insulin increases considerably owing to the effect of dexamethasone on glucose metabolism.

Major surgery causes a stress reaction, which can contribute to anaerobic metabolism and inadequacy of tissue perfusion, leading to increased lactate levels. An association between increased lactate levels and increased morbidity and mortality has been shown in many studies. ${ }^{33,34}$ Patients in the DEX group had statistically significantly higher lactate levels for 3 days postoperatively $(P<.001)$. An increase in lactate levels could be related to increased glucose levels as mentioned by Ottens et $\mathrm{al}^{35}$ in their prospective trial. This increase will automatically lead to an

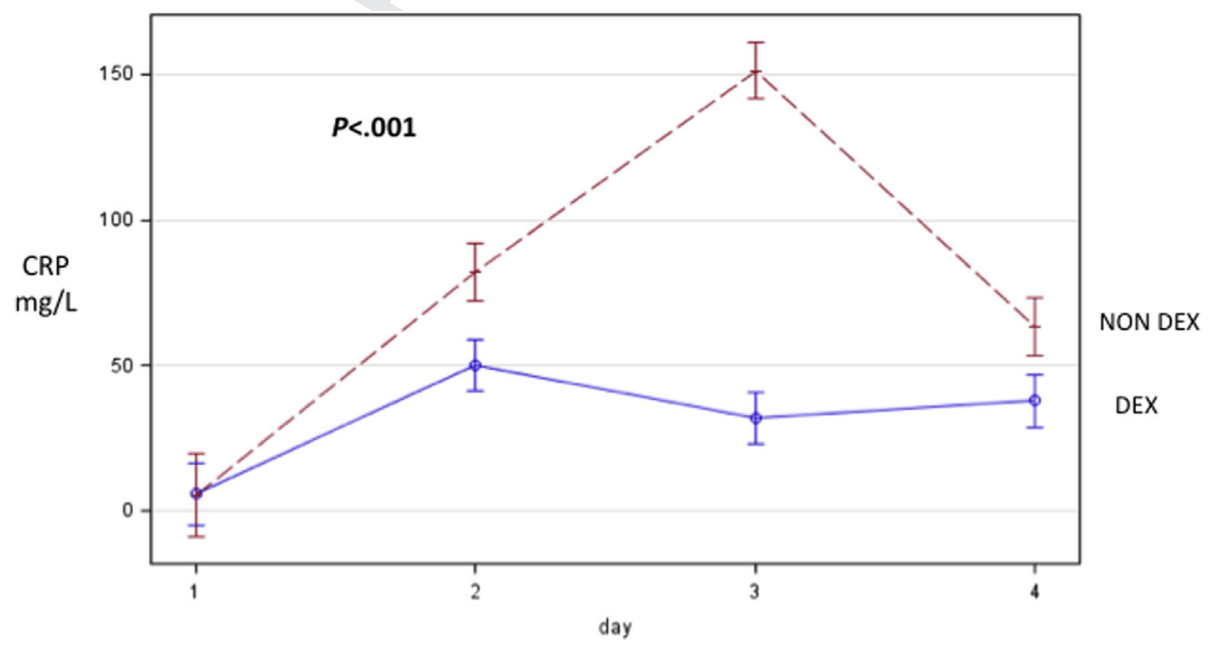

FIGURE 4. Serum CRP levels after microvascular reconstruction. Data are presented as means with $95 \%$ confidence limits. CRP, C-reactive protein; DEX, patients with dexamethasone; NON-DEX, patients without dexamethasone.

Kainulainen et al. Dexamethasone and Postoperative Recovery. J Oral Maxillofac Surg 2018. 


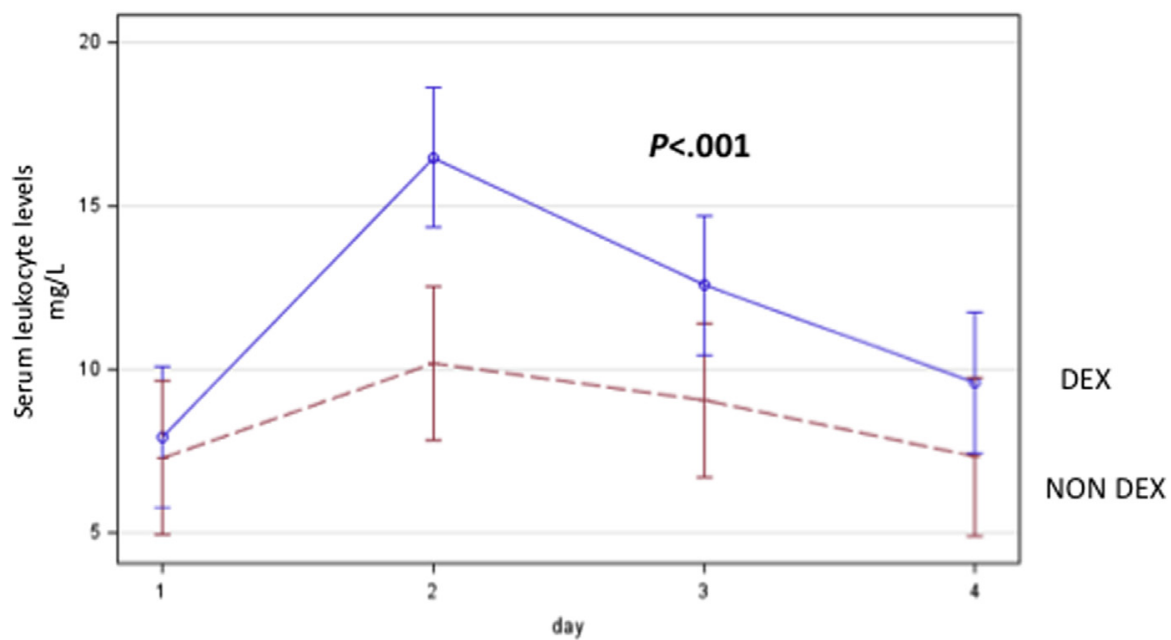

FIGURE 5. Serum leukocyte levels after microvascular reconstruction. Data are presented as means with $95 \%$ confidence limits. DEX, patients with dexamethasone; NON-DEX, patients without dexamethasone.

Kainulainen et al. Dexamethasone and Postoperative Recovery. J Oral Maxillofac Surg 2018.

increased amount of insulin, as seen in the present study. Although lactate levels were statistically higher in patients in the DEX group, the levels were relatively low (0 to $2.2 \mathrm{mmol} / \mathrm{L}$ ) and within normal limits. Lactate was not a prognostic factor in this study when considering immediate recovery.

Trauma and surgery change blood counts. GCs decrease the systemic inflammatory response caused by surgical trauma. Use of preoperative and perioperative GCs is associated with postoperative leukocytosis and lower CRP levels. ${ }^{36,37}$ Postoperative leukocyte and CRP concentrations are useful markers of the magnitude of operative injury. ${ }^{38,39}$ In the present study, CRP levels also were considerably lower and leukocyte counts were markedly higher as was expected in patients receiving dexamethasone. In particular, the low CRP values (caused by dexamethasone) might cause doctors to overlook early-onset infections.

Despite being the largest prospective randomized double-blinded trial of patients with reconstructive surgery for head and neck cancer and perioperative use of dexamethasone, the total number of patients could have been larger. However, the number of patients undergoing complex ablative and reconstructive surgery is limited even in a tertiary university center. The present results have already decreased the perioperative use of dexamethasone in reconstructive head and neck cancer surgery in Finland.

In this study, the only benefit of perioperative dexamethasone use was the lower total dose of oxycodone. However, the disadvantages were greater; these included the need for increased insulin, disturbed sugar metabolism, higher lactate levels, and misleading CRP values. Dexamethasone is used quite liberally in reconstructive head and neck surgery.
The use of perioperative dexamethasone in patients head and neck cancer and microvascular reconstruction seems to increase the risk of postoperative infection. ${ }^{20}$ Although dexamethasone decreases postoperative pain, the routine use of dexamethasone is not justified in this patient group because it does not seem to meaningfully decrease nausea, hasten rehabilitation, or shorten ICU or hospital stay.

\section{References}

1. Hurvitz KA, Kobayashi M, Evans GR: Current options in head and neck reconstruction. Plast Reconstr Surg 118:122e, 2006

2. Wong CH, Wei FC: Microsurgical free flap in head and neck reconstruction. Head Neck 32:1236, 2010

3. Rinaldo A, Shaha AR, Wei WI, et al: Microvascular free flaps: A major advance in head and neck reconstruction. Acta Otolaryngol 122:779, 2002

4. Diakos EA, Gallos ID, El-Shunnar S, et al: Dexamethasone reduces pain, vomiting and overall complications following tonsillectomy in adults: A systematic review and meta-analysis of randomised controlled trials. Clin Otolaryngol 36:531, 2011

5. Karanicolas PJ, Smith SE, Kanbur B, et al: The impact of prophylactic dexamethasone on nausea and vomiting after laparoscopic cholecystectomy: A systematic review and meta-analysis. Ann Surg 248:751, 2008

6. De Oliveira GS Jr, Castro-Alves LJ, Ahmad S, et al: Dexamethasone to prevent postoperative nausea and vomiting: An updated meta-analysis of randomized controlled trials. Anesth Analg 116: 58,2013

7. Dan AE, Thygesen TH, Pinholt EM: Corticosteroid administration in oral and orthognathic surgery: A systematic review of the literature and meta-analysis. J Oral Maxillofac Surg 68: 2207,2010

8. Kormi E, Snall J, Tornwall J, Thoren H: A survey of the use of perioperative glucocorticoids in oral and maxillofacial surgery. J Oral Maxillofac Surg 74:1548, 2016

9. Waldron NH, Jones CA, Gan TJ, et al: Impact of perioperative dexamethasone on postoperative analgesia and side-effects: Systematic review and meta-analysis. Br J Anaesth 110:191, 2013

10. Henzi I, Walder B, Tramer MR: Dexamethasone for the prevention of postoperative nausea and vomiting: A quantitative systematic review. Anesth Analg 90:186, 2000 
11. Fleming PS, Flood TR: Steroid-induced psychosis complicating orthognathic surgery: A case report. Br Dent J 199:647, 2005

12. Chan MH, Chan PK, Griffith JF, et al: Steroid-induced osteonecrosis in severe acute respiratory syndrome: A retrospective analysis of biochemical markers of bone metabolism and corticosteroid therapy. Pathology 38:229, 2006

13. Hussain A, Young WB: Steroids and aseptic osteonecrosis (AON) in migraine patients. Headache 47:600, 2007

14. O'Neil EA, Chwals WJ, O'Shea MD, Turner CS: Dexamethasone treatment during ventilator dependency: Possible life threatening gastrointestinal complications. Arch Dis Child 67(1 Spec No):10, 1992

15. Mastropietro CW, Barrett R, Davalos MC, et al: Cumulative corticosteroid exposure and infection risk after complex pediatric cardiac surgery. Ann Thorac Surg 95:2133, 2013

16. Percival VG, Riddell J, Corcoran TB: Single dose dexamethasone for postoperative nausea and vomiting-A matched case-control study of postoperative infection risk. Anaesth Intensive Care 38: 661,2010

17. Snall J, Kormi E, Koivusalo AM, et al: Effects of perioperatively administered dexamethasone on surgical wound healing in patients undergoing surgery for zygomatic fracture: A prospective study. Oral Surg Oral Med Oral Pathol Oral Radiol 117:685, 2014

18. Kainulainen S, Tornwall J, Koivusalo AM, et al: Dexamethasone in head and neck cancer patients with microvascular reconstruction: No benefit, more complications. Oral Oncol 65:45, 2017

19. Watcha MF, White PF: Postoperative nausea and vomiting. Its etiology, treatment, and prevention. Anesthesiology 77:162, 1992

20. Capes SE, Hunt D, Malmberg K, Gerstein HC: Stress hyperglycaemia and increased risk of death after myocardial infarction in patients with and without diabetes: A systematic overview. Lancet 355:773, 2000

21. Pittas AG, Siegel RD, Lau J: Insulin therapy and in-hospital mortality in critically ill patients: Systematic review and metaanalysis of randomized controlled trials. J Parenter Enteral Nutr 30:164, 2006

22. Griesdale DE, de Souza RJ, van Dam RM, et al: Intensive insulin therapy and mortality among critically ill patients: A metaanalysis including NICE-SUGAR study data. CMAJ 180:821, 2009

23. Afman CE, Welge JA, Steward DL: Steroids for post-tonsillectomy pain reduction: Meta-analysis of randomized controlled trials. Otolaryngol Head Neck Surg 134:181, 2006

24. Nielsen RV, Siegel H, Fomsgaard JS, et al: Preoperative dexamethasone reduces acute but not sustained pain after lumbar disk surgery: A randomized, blinded, placebo-controlled trial. Pain 156:2538, 2015

25. Baxendale BR, Vater M, Lavery KM: Dexamethasone reduces pain and swelling following extraction of third molar teeth. Anaesthesia 48:961, 1993
26. Kawakami H, Mihara T, Nakamura N, et al: Effect of an intravenous dexamethasone added to caudal local anesthetics to improve postoperative pain: A systematic review and metaanalysis with trial sequential analysis. Anesth Analg 125:2072, 2017

27. Widar F, Kashani H, Alsen B, et al: The effects of steroids in preventing facial oedema, pain, and neurosensory disturbances after bilateral sagittal split osteotomy: A randomized controlled trial. Int J Oral Maxillofac Surg 44:252, 2015

28. Clayburgh D, Stott W, Bolognone R, et al: A randomized controlled trial of corticosteroids for pain after transoral robotic surgery. Laryngoscope 127:2558, 2017

29. Wattwil M, Thorn SE, Lovqvist A, et al: Dexamethasone is as effective as ondansetron for the prevention of postoperative nausea and vomiting following breast surgery. Acta Anaesthesiol Scand 47:823, 2003

30. Jahromi HE, Gholami M, Rezaei F: A randomized double-blinded placebo controlled study of four interventions for the prevention of postoperative nausea and vomiting in maxillofacial trauma surgery. J Craniofac Surg 24:e623, 2013

31. Furst SR, Rodarte A: Prophylactic antiemetic treatment with ondansetron in children undergoing tonsillectomy. Anesthesiology 81:799, 1994

32. Dieleman JM, Nierich AP, Rosseel PM, et al: Intraoperative high-dose dexamethasone for cardiac surgery: A randomized controlled trial. JAMA 308:1761, 2012

33. Hajjar LA, Almeida JP, Fukushima JT, et al: High lactate levels are predictors of major complications after cardiac surgery. $J$ Thorac Cardiovasc Surg 146:455, 2013

34. Kaukonen KM, Bailey M, Egi M, et al: Stress hyperlactatemia modifies the relationship between stress hyperglycemia and outcome: A retrospective observational study. Crit Care Med 42:1379, 2014

35. Ottens TH, Nijsten MW, Hofland J, et al: Effect of high-dose dexamethasone on perioperative lactate levels and glucose control: A randomized controlled trial. Crit Care 19:41, 2015

36. McSorley ST, Horgan PG, McMillan DC: The impact of preoperative corticosteroids on the systemic inflammatory response and postoperative complications following surgery for gastrointestinal cancer: A systematic review and meta-analysis. Crit Rev Oncol Hematol 101:139, 2016

37. Demura S, Takahashi K, Murakami H, et al: The influence of steroid administration on systemic response in laminoplasty for cervical myelopathy. Arch Orthop Trauma Surg 133:1041, 2013

38. Watt DG, Horgan PG, McMillan DC: Routine clinical markers of the magnitude of the systemic inflammatory response after elective operation: A systematic review. Surgery 157:362, 2015

39. Deirmengian GK, Zmistowski B, Jacovides C, et al: Leukocytosis is common after total hip and knee arthroplasty. Clin Orthop Relat Res 469:3031, 2011 